\title{
LA ATENCIÓN SELECTIVA MODULA EL PROCESAMIENTO DE LA INFORMACIÓN Y LA MEMORIA IMPLÍCITA SELECTIVE ATTENTION MODULATES INFORMATION PROCESSING AND IMPLICIT MEMORY
}

\author{
SoledAd BALlesteros
}

Cómo referenciar este artículo/How to reference this article:

Ballesteros, S. (2014). La atención selectiva modula el procesamiento de la información y la memoria implícita [Selective attention modulates information processing and implicit memory]. Acción Psicológica, 11(1), 7-20. http://dx.doi.org/10.5944/ap.1.1.13788

\section{Resumen}

La atención selectiva permite procesar información relevante mientras suprime la irrelevante que puede aparecer simultáneamente en el campo visual aunque con frecuencia el observador no puede ignorar la información irrelevante. En esta revisión discutimos resultados obtenidos a partir de la tarea Stroop con palabras de color fragmentadas como estímulos y otros resultados que muestran que la variación de una dimensión irrelevante en una tarea visual igual-diferente como dos ejemplos de que dimensiones estimulares irrelevantes para la tarea, producen interferencia. Finalmente, describimos los resultados de varios estudios diseñados para investigar el efecto de la atención durante la codificación del estímulo en la memoria implícita evaluada por la existencia de priming de repetición a lo largo del ciclo vital. Encontramos priming para los estímulos atendidos en los niños, adultos jóvenes y mayores sanos pero no para los estímulos presen- tados durante la codificación pero no atendidos. Sin embargo, no encontramos priming para los objetos atendidos ni para los no atendidos en mayores con déficit cognitivo leve y en enfermos de Alzheimer. Los resultados sugieren: (1) que la memoria implícita no es automática y requiere atención; y (2) la existencia de un déficit temprano de la atención selectiva como posible marcador del envejecimiento patológico.

Palabras clave: Atención selectiva; Dimensiones integrales-separables; Efecto Stroop; Enfermedad de Alzheimer; Envejecimiento; Memoria Implícita.

\begin{abstract}
Selective attention allows to process relevant stimuli while suppressing irrelevant ones that appear simultaneously in the visual field. However, many times the perceiver cannot ig-
\end{abstract}

Correspondencia: Departamento de Psicología Básica II. Facultad de Psicología. UNED. C/ Juan del Rosal, 10. 28040 Madrid. Tfno.: 91 3986227. Email: mballesteros@psi.uned.es 
nore irrelevant information. In this review, I discussed results from the Stroop task with fragmented colour word stimuli as well as other findings showing that the variation of an irrelevant dimension on same-different visual judgments as two examples that irrelevant stimulus dimensions produced interference. Finally, I describe the results of several studies designed to investigate the effect of selective attention at encoding on implicit memory across lifespan. Priming for attended stimuli at encoding was found for children, young adults and healthy older adults. Priming was not found for unattended stimuli suggesting that implicit memory requires attention at encoding. Alzheimer's patients and cognitively impaired older adults did not show priming for attended or for unattended stimuli. The findings suggest that: (1) implicit memory is not automatic and requires attention at encoding; and (2) an early deficit of selective attention might be a marker of pathological aging.

Keywords: Aging, Alzheimer's disease; Implicit memory; Integral-separable dimensions; Selective attention; Stroop effect.

\section{Influencia de la información irrelevante en percepción, atención y memoria}

\section{Introducción}

Las personas perciben, comparan y recuerdan patrones visuales y objetos mientras tratan de ignorar información irrelevante que acompaña a la información relevante. Parece fácil comparar dos estímulos en una determinada dimensión cuando concurrentemente aparece información irrelevante. Sin embargo, el perceptor frecuentemente no puede dejar de atender a la información irrelevante, lo que produce retraso en la respuesta. Una pregunta relevante para los investigadores durante décadas, incluidos el Dr. Manga y la autora de este artículo, fue si el tipo de dimensiones estimulares que se seleccionen como la dimensión relevante o irrelevante influye en la estructura percibida (Ballesteros, 1989; Ballesteros y Manga, 1994, 1996). En esta revisión, se describen resultados experimentales que muestran que la información no atendida puede interferir en la respuesta pero a la vez, paradójicamente, mostraremos cómo los estímulos presentes pero no atendidos, no se codifican en la memoria.

La atención desempeña un papel central en la memoria episódica (explícita, consciente) porque es necesaria para que se formen trazas de memoria duraderas (Ballesteros, Reales, García y Carrasco, 2006; Rock y Gutman, 1981). El papel de la atención en la memoria implícita ha sido más discutido. La memoria implícita es un tipo de memoria inconsciente (involuntaria) que se evalúa mediante la existencia de priming de repetición (mejor actuación con los estímulos repetidos que con los nuevos). Sin embargo, los resultados de un número cada vez mayor de estudios sugieren que la memoria implícita para estímulos verbales y no verbales presentados visualmente, y de objetos presentados a través del tacto, requiere atención durante la fase de codificación. Estudios realizados con niños (Ballesteros, Reales y García, 2007), adultos jóvenes (Ballesteros et al., 2006), mayores sanos, enfermos de Alzheimer (Ballesteros, Reales, Mayas y Heller, 2008) y mayores con déficit cognitivo leve (Ballesteros, Mayas y Reales, 2013) sugieren que independientemente de la edad, la condición y la modalidad a la que presenten los estímulos, la memoria implícita no es automática y requiere atención durante la codificación estimular. Las personas jóvenes y sanas muestran memoria implícita para los estímulos atendidos pero la falta de esta memoria para los estímulos atendidos podría ser un marcador de envejecimiento patológico. Finalmente, concluiremos señalando que la neuroplasticidad, la adaptación y el reclutamiento de zonas cerebrales adicionales a las utilizadas por los jóvenes podría ser la forma que tiene el cerebro del mayor para adaptarse a los cambios producidos con la edad. La realización de actividades estimulantes, el ejercicio físico y el entrenamiento con videojuegos pueden contribuir a mantener la vitalidad 
cognitiva en los mayores, retrasando los efectos negativos del envejecimiento patológico.

\section{¿Influye la información irrelevante en la percepción?}

La atención selectiva permite al perceptor procesar estímulos relevantes mientras suprime el procesamiento de estímulos irrelevantes para la tarea que pueden aparecer simultáneamente en el campo visual junto a los relevantes. Sin embargo, el observador humano no puede en muchas ocasiones ignorar la información irrelevante (Ballesteros y Manga, 1996).

Una de las tareas más utilizadas en psicología cognitiva es la tarea de Stroop (Stroop, 1935) que después de casi un siglo sigue interesando a los investigadores porque incide en operaciones perceptivas muy primitivas (ver MacLeod, 1991) y porque se trata de un efecto robusto fácilmente replicable en el laboratorio y en la clínica. Cuando se pide a las personas que nombren el color de la tinta en la que aparece escrita una palabra que se refiere a un color (la palabra «ROJO» escrita en tinta azul), la respuesta aparece inhibida (condición incongruente) y requiere más tiempo que cuando la palabra que denota un color aparece escrita en ese mismo color (condición congruente). Para explicar el efecto de la interferencia Stroop que produce la dimensión irrelevante (la palabra de color) sobre el nombrado del color se han propuesto distintas teorías pero ninguna de las propuestas hasta ahora han podido explicar todos los resulta- dos empíricos encontrados. El efecto Stroop se define como el aumento del tiempo de respuesta ante los ensayos incongruentes en comparación con estímulos neutros (XXXX de color rojo, por ejemplo). Numerosas conductas reflejas se dice que son automáticas porque ocurren sin intención. Otras conductas sobre-aprendidas como la lectura son automáticas porque el individuo no puede sustraerse a la lectura de las palabras como ocurre en la tarea Stroop.

El efecto Stroop es un claro ejemplo de que dimensiones estimulares irrelevantes para la tarea, se procesan en cierta medida porque el perceptor no puede dejar de atenderlas. Esto se traduce en mayor tiempo de respuesta para indicar el color en el que está escrita la palabra (de color). Se dice que la lectura de la palabra es automática y el perceptor no puede dejar de leerla porque se trata de una actividad inconsciente e involuntaria a la que no puede sustraerse a pesar de que las instrucciones indican explícitamente nombrar el color (Reisberg, 1997).

La manipulación del nivel de fragmentación del estímulo permite estudiar cómo influye la cantidad de información contenida en el estímulo en la interferencia y facilitación Stroop. En una serie de estudios (Ballesteros, Reales y Manga, 2000) manipulamos el nivel de fragmentación de la palabra desde el nivel más fragmentado (nivel 1) hasta el nivel 8 (palabra completa) utilizando el algoritmo de Snodgrass y Poster (1992). La Figura 1 presenta ejemplos de estos niveles en la condición congruente, incongruente y neutral. 


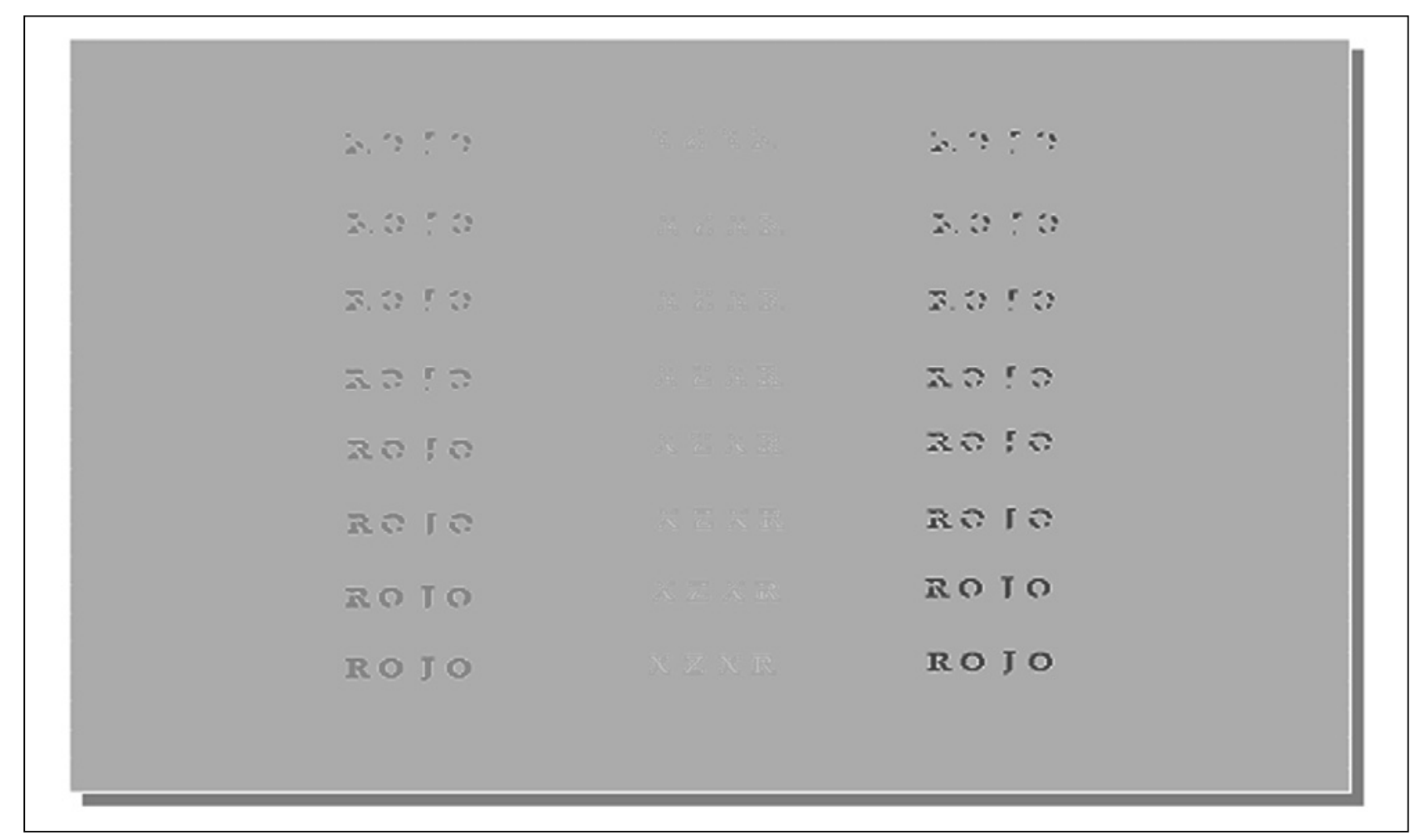

Figura 1. Ejemplos de los 8 niveles de fragmentación de los estímulos de color de arriba, el nivel más fragmentado (nivel 1) hasta abajo, el nivel más completo (nivel 8) en la condición Congruente (izquierda), Neutral (centro) e Incongruente (derecha)

Los resultados mostraron que cuando se presentaban las palabras a diferentes niveles de fragmentación bloqueadas en función de las diferentes condiciones (estímulos congruentes, incongruentes, neutros presentados en bloques experimentales diferentes) aparecía interferencia Stroop en la condición incongruente y facilitación en la congruente en todos los niveles de fragmentación. Sin embargo, cuando la presentación de los estímulos fue aleatorizada (estímulos congruentes, incongruentes y neutros presentados entremezclados al azar), la interferencia Stroop apareció a partir del nivel de fragmentación 4 (al que ya empezaba a leerse la palabra), y aumentó hasta el nivel de fragmentación 8 (palabra completa). Estos resultados sugieren la existencia de una predisposición o set de respuesta que produce interferencia en niveles de fragmentación en los que no se puede leer la palabra de color y, por tanto, el perceptor no puede todavía procesar la información semántica. Parece que las condiciones congruentes e incongruentes crean un efecto contextual en el que palabras o estímulos que pueden leerse mejor influyen en las expectativas para responder a palabras menos legibles. Por el contrario, cuando no existen esas expectativas, la interferencia aparece solo cuando el estímulo puede empezar a leerse. En un estudio electroencefalográfico reciente hemos investigado el efecto de la fragmentación a los estímulos de color usando potenciales evocados (Ballesteros, Muñoz y Reales, 2013). 


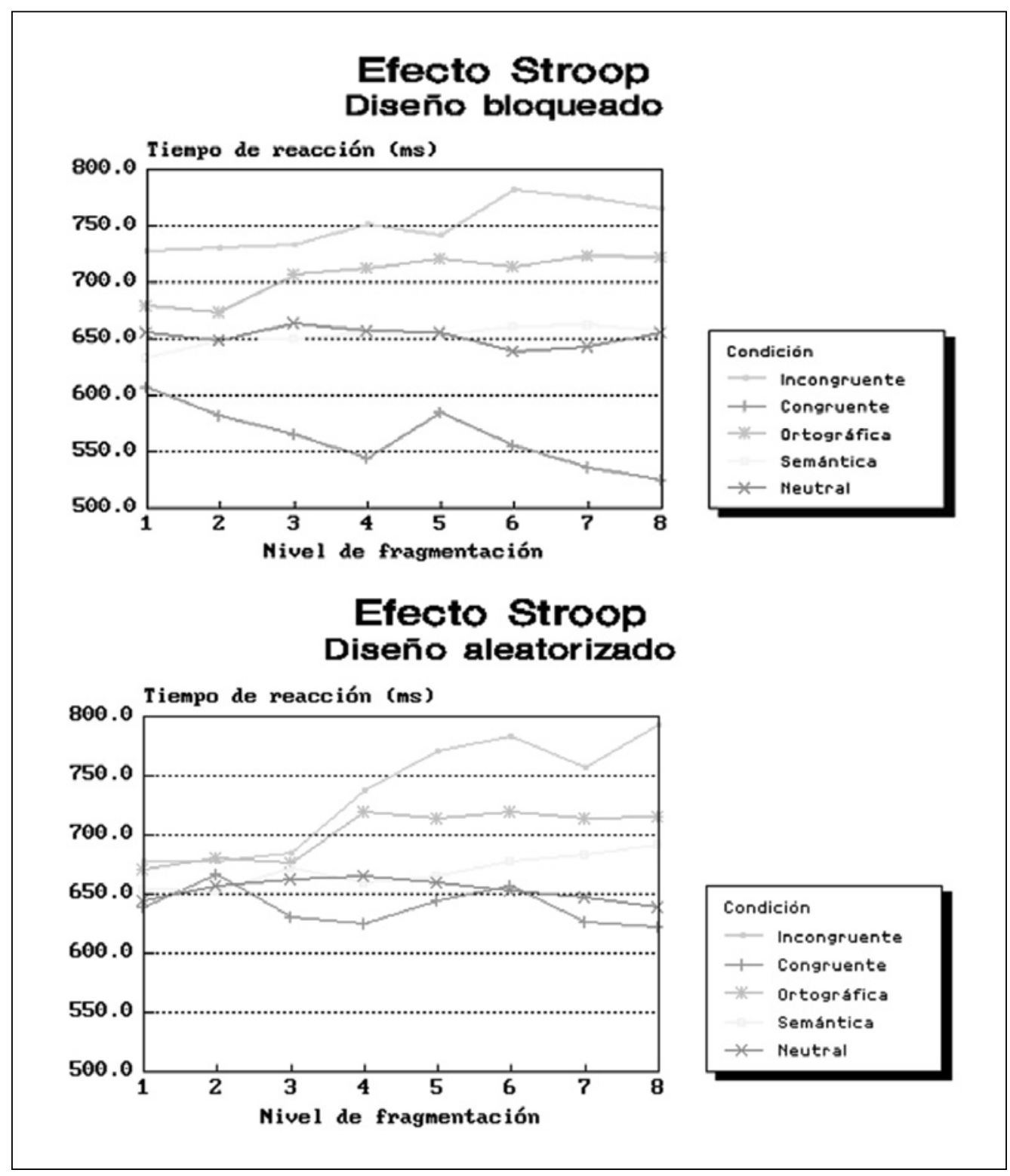

Figura 2. Efectos de facilitación e interferencia en varias condiciones experimentales en función del nivel de fragmentación del estímulo (del nivel 1 al nivel 8). Se muestran los resultados obtenidos con un diseño de presentación estimular bloqueado (arriba) y aleatorizado (abajo). A partir de Ballesteros, Reales y Manga (2010), Psicothema, 12, Supl. n. ${ }^{\circ} 2$

Otra forma de estudiar la influencia de información irrelevante en la clasificación rápida de patrones visuales multidimensionales (Ballesteros y Manga, 1994, 1996) consiste en comparar rápidamente patrones que varían en varios niveles en dos dimensiones diferentes. En varios estudios nos preguntamos cómo afecta la información irrelevante en tareas de comparación de patrones visuales que variaban en distintos niveles de cada dimensión. Dixon y Just (1978) habían propuesto el modelo de la normalización según el cual, el sistema visual realiza una transformación continua igualando primero los dos patrones en la dimen- 
sión irrelevante para la tarea de clasificación «igual-diferente», para después compararlos en la dimensión relevante. La principal predicción de este modelo es que los tiempos de reacción (TRs) de los juicios «igual» $\mathrm{y}$ «diferente» mostraran una tendencia lineal creciente en función del número de niveles de discrepancia existentes en la dimensión irrelevante. Sin embargo, esta explicación transformacional no ha sido totalmente confirmada por los datos, especialmente en los juicios «diferente».

La hipótesis puesta a prueba por Ballesteros y Manga (1996) fue que la dimensión irrelevante elegida importaba, y especialmente su lugar en el continuo integralidad-separabilidad. En varios experimentos comprobamos si los TRs aumentaban linealmente con distintos niveles de variación en la dimensión irrelevante para respuestas «igual» $\mathrm{y}$ «diferente». Los estímulos fueron elipses que variaban en 4 niveles de inclinación (45, 90, 135 y 180 grados comenzando desde la posición horizontal) y en 4 niveles de tamaño (eje mayor de las elipses $1.4,1.8,2.2$, y $2.6 \mathrm{~cm}$, y eje menor $0.8,1.0$, 1.2 y $1.4 \mathrm{~cm}$ ). La inclinación y el tamaño son dimensiones situadas más próximas al extremo separable del continuo integralidad-separabilidad (Ballesteros, 1989). Los resultados, tanto cuando la dimensión relevante fue tamaño de las elipses (Experimento 1) como cuando fue orientación (Experimento 2) correspondientes a las respuestas «igual» mostraron que los TRs aumentaban con el aumento de la disparidad (número de niveles) aunque la tendencia lineal no fue significativa. El tiempo necesario para procesar la orientación estuvo influido por la magnitud de la disparidad irrelevante en el tamaño de las elipses, lo que concuerda con la propuesta de transformación mental. Sin embargo, los resultados de los juicios «diferente» fueron contrarios a las predicciones. Cuando la dimensión relevante fue $t a-$ maño, cuanto mayor fue la disparidad en orientación más rápida fue la respuesta. Cuando lo fue la orientación, los distintos niveles de disparidad en tamaño no influyeron en los TRs. Estos resultados no apoyan la hipótesis de la normalización. En los siguientes experimentos utilizamos 16 rectángulos del mismo tamaño que variaban en brillo y orientación.
Los rectángulos se construyeron a partir de la combinación ortogonal de 4 niveles de brillo y 4 niveles de orientación. La predicción fue que el aumento en brillo no interfiriera con el procesamiento de la orientación de los rectángulos porque el brillo tiene un grado de integralidad bajo cuando se combina con otras dimensiones. Los participantes juzgaron si dos rectángulos del mismo tamaño eran iguales o diferentes en brillo (Experimento 3) o en orientación (Experimento 4). En los juicios «igual» no encontramos efectos. En los juicios «diferente» se produjo una disminución (juicios de brillo) o un aumento (juicios de orientación) en los TRs con la disparidad.

Estos resultados mixtos no concuerdan con las predicciones de la hipótesis de la normalización. Con dimensiones más separables como brillo y orientación, la normalización no fue necesaria ni siquiera para los juicios «igual». Los resultados sugieren que el tipo de dimensiones utilizadas para construir las formas visuales influyen en la estructura percibida (Garner, 1974; Ballesteros, 1989; Ballesteros y Manga, 1996).

\section{La atención selectiva modula la memoria implícita y explícita a lo largo del ciclo vital}

Mientras nadie duda de la importancia de la atención en la memoria explícita (i.e., Craik, Goboni, Naveh-Benjamin y Anderson, 1996; Mulligan, 1998; Rock y Gutman, 1981), su papel en la memoria implícita ha sido más discutido. Algunos autores sugirieron que la atención selectiva durante la codificación de los estímulos no influía en la memoria implícita o, en todo caso, el efecto era pequeño (e.g., Parkin y Russo, 1990). El paradigma de atención dividida utilizado tiene varias limitaciones metodológicas ya que un ritmo de presentación lento permite al participante dirigir su atención de la información atendida a la no atendida, facilitando la codificación en la memoria implícita (Crabb y Dark, 1999). Además, los estudios que no han encontrado efecto de la atención han utilizado modalidades diferentes (audición y visión) para presentar la informa- 
ción atendida y no atendida. Estudios realizados con material verbal han mostrado efectos atencionales tanto en la memoria explícita como en la implícita (Crabb y Dark, 1999; MacDonald y MacLeod, 1998).

En nuestro laboratorio hemos estudiado el papel de la atención en la memoria implícita de dibujos y objetos, encontrando que la atención selectiva durante la codificación de los estímulos influye en ambos tipos de memoria aunque según la prueba utilizada para evaluar la memoria implícita puede influir menos que en la memoria explícita (Ballesteros et al., 2006; Ballesteros et al., 2007; Ballesteros et al., 2008; Ballesteros et al., 2013). Estos estudios realizados con distintos grupos de edad, características, formas de presentación del estímulo atendido y no atendido y pruebas utilizadas para evaluar la memoria implícita, sugieren que la memoria implícita no es automática y requiere atención durante la fase de codificación. En los siguientes apartados revisaremos los principales resultados obtenidos en una serie de estudios realizados con niños, adultos jóvenes, mayores sanos y con envejecimiento patológico.

\section{1 ¿Influye la atención selectiva en la memoria de los adultos jóvenes y de los niños?}

En un estudio realizado con jóvenes (Ballesteros et al., 2006) combinamos un paradigma de priming de repetición con un procedimiento de atención selectiva durante la fase de codificación y encontramos que la atención es una variable importante no solo para codificar información y recuperarla después voluntariamente a partir de la memoria episódica, sino también de forma involuntaria (implícita). Durante la fase de estudio presentamos en cada ensayo en el centro de la pantalla del ordenador dos dibujos lineales solapados, uno de color verde y el otro azul (ver Figura 3, izquierda) mientras los participantes nombraban rápidamente el objeto de color verde (objeto atendido). Los dibujos lineales se seleccionaron del conjunto de Snodgrass y Vanderwart (1980). Después de 5 minutos de tarea distractora, diferentes grupos de participantes realizaron de manera incidental la prueba de Compleción de fragmentos dibujos para evaluar su memoria implícita (Experimentos 1 y 2, ver Figura 4) o la de Denominación rápida de objetos (Experimento 3).

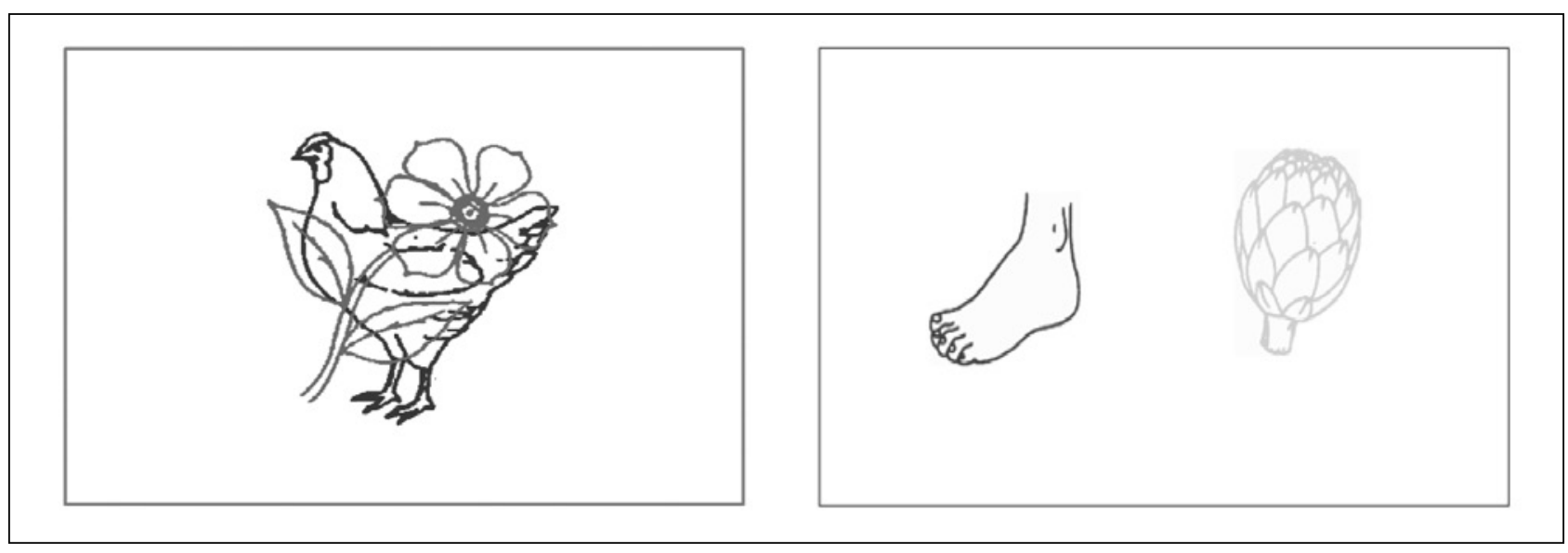

Figura 3. Fase de estudio: La Figura muestra dos formas de presentar los estímulos (atendido y no atendido) durante la fase de codificación. A la izquierda se muestra un ejemplo de los dos dibujos lineales solapados presentados en el centro de la pantalla del ordenador. A la derecha se presenta otra forma de presentación en la que los estímulos se presentan a la derecha y a la izquierda del punto de fijación situado en el centro de la pantalla. El estímulo atendido fue el de color verde 


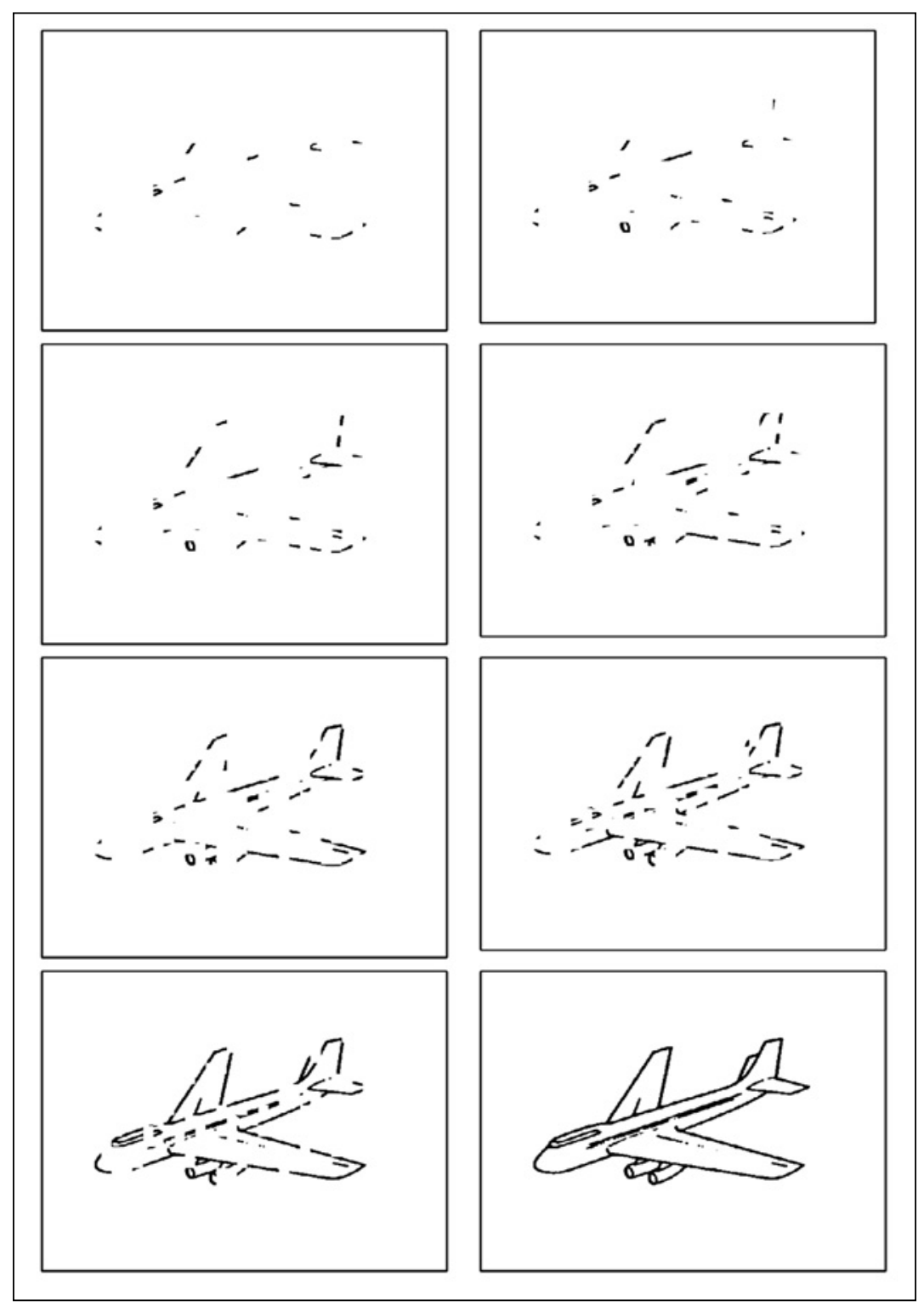

Figura 4. La Figura muestro los 8 niveles de fragmentación de los dibujos (desde el nivel más fragmentado (arriba izquierda) al más completo (abajo derecha) utilizados en la prueba de memoria implícita de Compleción de fragmentos de dibujos

En la prueba de Compleción de fragmentos, los estímulos fueron fragmentos de los dibujos presentados en negro sobre un fondo blanco. En esta fase se presentaron dibujos atendidos y no atendidos durante la fase de estudio y dibujos nuevos, todos ellos en un orden aleatorio. Cada ensayo comenzaba con la presentación de un dibujo al máximo nivel de fragmentación (nivel 1, ver Figura 4), si en 1.5 segundos, el participante no respondía aparecía en la pantalla el dibujo al nivel 2 de fragmentación y así hasta que respondiera, o hasta llegar al nivel 8 (dibujo completo). Al finalizar la prueba de memoria implícita, los participantes realizaron una prueba de memoria explícita, que según los experimentos consistió en una prueba de reconocimiento «antiguo-nuevo» o en una prueba de recuerdo libre. 
Los resultados mostraron que el umbral de identificación fue menor para los estímulos atendidos que para los no atendidos. Además, los dibujos atendidos se recordaron (Experimento 1) y se reconocieron (Experimentos 2 y 3) mejor que los no atendidos. Los participantes utilizaron el color como clave para seleccionar los dibujos lineales solapados. Los resultados mostraron que la atención selectiva durante la codificación produjo mejor representación del estímulo. Los estímulos atendidos durante la fase de codificación se procesaron más eficientemente que los estímulos del mismo tipo no atendidos que ocupaban una misma posición espacial durante la fase de codificación. La memoria implícita es menos sensible a la limitación de los recursos atencionales que la memoria explícita pero ni un tipo de memoria ni otro funcionan sin atención (Ballesteros et al., 2006).

La investigación con jóvenes había mostrado una mejor memoria implícita y mejor recuerdo y reconocimiento (medidas de memoria explícita) para los dibujos atendidos que para los no atendidos. Además, niños con déficit de atención y niños controles sanos de 8 y 11 años mostraron priming intacto para los estímulos atendidos pero no para los estímulos presentados durante la fase de codificación pero que no habían sido atendidos (Ballesteros et al., 2007).

\section{2 aModula la atención selectiva la memoria implícita en el envejecimiento normal y patológico?}

Numerosos estudios han mostrado los declives que se producen en el sistema cognitivo humano durante el envejecimiento. La velocidad de procesamiento de la información (Ballesteros et al., 2013; Verhaeghen y Cerella, 2008), la función ejecutiva, la memoria de trabajo (Braver y West, 2008) y la memoria episódica se deterioran con la edad pero otras funciones como las habilidades verbales, los conocimientos adquiridos o la memoria implícita no cambian en la vejez (Goh, An y Resnick, 2012; Osorio, Fay, Pouthas y Ballesteros, 2010; Park y Reuter-Lorenz, 2009). Numerosos estudios conductuales que han utilizado diferentes tareas para evaluar la memoria implícita han encontrado que este tipo de memoria está preservada en mayores sanos (Ballesteros et al., 2008, 2009; Ballesteros, Bischof, Goh, y Park, 2013; Wiggs, Weisberg y Martin, 2006), en enfermos de Alzheimer (EA) (Ballesteros y Reales, 2004; Ballesteros, Reales y Mayas, 2007; Fleischman, 2007), e incluso cuando se cambia de modalidad de la fase de estudio (tacto) a la fase de prueba de memoria (visión), como ocurre en la memoria implícita crosmodal (Ballesteros, González, Mayas, Reales, y García, 2009).

¿Los mayores sanos y los EA mostrarán el mismo patrón que los niños y los adultos jóvenes cuando se compromete su atención selectiva? ¿Su actuación disminuirá con la edad y con la enfermedad? ¿Hará falta la misma atención selectiva cuando los objetos se procesan visualmente que cuando se presentan al tacto? En un estudio en el que participaron adultos jóvenes, mayores sanos y pacientes de Alzheimer intentamos responder a estas preguntas (Ballesteros et al., 2008). El procedimiento utilizado en el estudio visual consistió en una fase de estudio similar a los estudios previos con niños y adultos. En la fase de prueba, los participantes realizaron de manera incidental una prueba de Compleción de dibujos. Los jóvenes y mayores sanos identificaron los dibujos atendidos durante la fase de estudio a un nivel más fragmentado que los no atendidos y los nuevos (no estudiados). Es importante señalar que el grupo EA no mostró facilitación ni para los estímulos ni para los no atendidos.

Para comprobar si hace falta la misma atención selectiva cuando los objetos se procesan visualmente que cuando se presentan al tacto sin visión, realizamos otro experimento en el que participaron grupos diferentes de jóvenes, de mayores sanos y EA. Durante la fase de estudio, la atención se dirigió hacia el objeto sujetado con la mano derecha o con la mano izquierda, nombrando lo antes posible el objeto mantenido en la mano atendida. Los estímulos fueron 48 objetos familiares (objetos del hogar, vegetales, herramientas, objetos para el cuidado personal) que no emitían olores ni hacían ruidos que permitieran su identificación. Estos objetos se distribuyeron en 6 cestas con 8 objetos cada una. Para evaluar la memoria implícita utilizamos el taquistoscopio háptico (Ballesteros $\mathrm{y}$ 
Reales, 2004; Reales y Ballesteros, 1999; Sebastián y Ballesteros, 2012). Se trata de un aparato de metacrilato que no permitía ver los objetos colocados en la plataforma de presentación que tenía un piezo-sensor debajo de la zona donde si situaba el objeto para ser explorado. Un programa registraba el tiempo de respuesta desde que el participante tocaba el objeto hasta que respondía a través de una llave vocal.

Durante la fase de estudio, el participante sentado delante de una mesa con los ojos tapados para no ver los objetos tenía delante dos cestas con 8 objetos familiares cada una (ver Figura 5A). En cada ensayo, tomaba dos objetos simultáneamente, uno con cada mano, y nombraba lo antes posible el de la mano atendida. Después, colocaba los dos objetos en dos cestas situadas detrás. Cada bloque de 8 ensayos, el experimentador dirigía la atención alternativamente a su mano izquierda o a su mano derecha. Después de cada bloque de 8 ensayos, las cestas vacías se sustituían por otras que contenían también 8 objetos cada una y la atención del participante se dirigía hacia la otra mano.

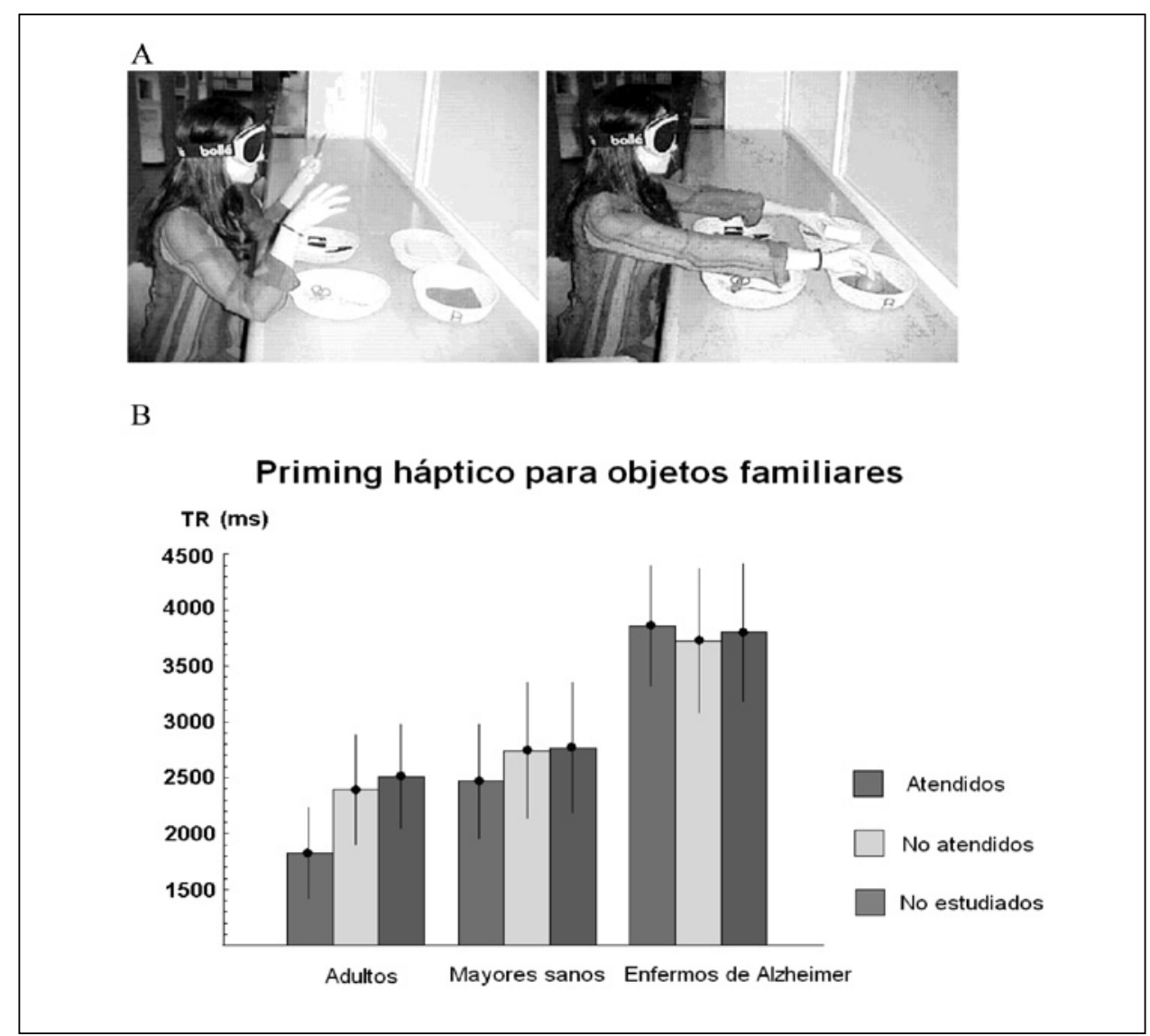

Figura 5 (A): En la fase de estudio el participante con los ojos tapados tomaba en cada ensayo un objeto con cada mano. En cada bloque de ensayos, el experimentador dirigía su atención hacia una mano y tenía que nombrar lo antes posible el objeto de la mano atendida y depositaba cada objeto en las cestas situadas en la parte posterior.

(B): Tiempos de respuesta en la tarea implícita de denominación rápida de objetos explorados a través del tacto (sin visión) correspondientes a los objetos atendidos durante la fase de estudio, no atendidos y nuevos. Jóvenes y mayores sanos muestran memoria implícita haptica para los objetos atendidos pero los enfermos de Alzheimer no [modificado a partir de Ballesteros, Reales, Mayas y Heller (2008), Experimental Brain Research, 1289, 56-68] 
Después de 5 minutos de tarea distractora, los participantes realizaron una prueba de $D e$ nominación rápida de objetos para evaluar su memoria implícita. En esta fase se presentaron los 48 objetos (16 atendidos, 16 no atendidos y 16 nuevos o no estudiados). En cada ensayo, el experimentador colocaba un objeto seleccionado al azar en la plataforma de presentación de estímulos del taquistoscopio háptico. Un sonido del ordenador indicaba que el objeto estaba en la plataforma. El ordenador registraba automáticamente el tiempo de respuesta desde que el participante tocaba el objeto hasta que emitía la respuesta verbal.

Los resultados mostraron que tanto los jóvenes como los mayores sanos mostraron priming para los objetos atendidos. Sin embargo, como ocurrió en el experimento visual, no mostraron priming para los objetos no atendidos (ver Figura 5B). Los objetos presentados durante la fase de estudio pero que no fueron atendidos, no se nombraron más rápido que los objetos nuevos incluidos durante la fase de prueba de memoria. Los EA no mostraron priming ni para los objetos atendidos ni para los no atendidos. La falta de facilitación para los objetos atendidos en los pacientes cuando su atención selectiva se comprometió durante la codificación sugiere la existencia de un trastorno de la atención en los EA. La falta de memoria implícita para los objetos atendidos en estos pacientes contrasta con la facilitación normal encontrada cuando se presenta solo un estímulo en cada ensayo en la fase de codificación (Ballesteros y Reales, 2004; Ballesteros et al., 2007; Fleischman, 2007).

En resumen, utilizando dos paradigmas diferentes diseñados para estudiar la influencia de la atención selectiva visual y háptica durante la codificación, en un caso con dibujos y en otro con objetos tridimensionales, encontramos memoria implícita para los objetos atendidos en jóvenes y mayores sanos pero no en los EA. La falta de priming para los estímulos atendidos en estos enfermos podría ser una marca del envejecimiento patológico (Ballesteros et al., 2008). La atención selectiva durante la codificación es necesaria para la memoria implícita, tanto en visión como en el tacto. Estos resultados son consistentes con los obteni- dos con niños en edad escolar (Ballesteros et al., 2007) y con adultos (Ballesteros et al., 2006). Los EA no mostraron priming ni para estímulos presentados a la visión ni para los presentados al tacto aunque otros estudios han mostrado memoria implícita intacta para estos enfermos cuando tenían que codificar un único estímulo, tanto en el tacto (Ballesteros y Reales, 2004) como en visión (Ballesteros et al., 2007).

\subsection{Los mayores con déficit cognitivo leve (DCL) ¿Se comportan como los mayores sanos o como los EA?}

Ballesteros, Mayas y Reales (2013) comprobaron que la actuación de mayores con déficit cognitivo leve (DCL) en una tarea de memoria implícita cuando se comprometía su atención se parecía a la de los EA que no mostraban facilitación perceptiva para los estímulos atendidos. El hecho de no encontrar priming para los estímulos atendidos sugiere que la atención empieza a deteriorarse en un estadio temprano del envejecimiento patológico. En el estudio participaron 20 mayores con DCL, 20 jóvenes y 20 mayores sanos que realizaron una batería de pruebas psicológicas. Después se evaluó su memoria implícita con la tarea de Compleción de fragmentos de dibujos para evaluar la existencia de facilitación para estímulos repetidos atendidos y no atendidos. En la fase de estudio, en lugar de presentar los dibujos solapados se presentó un dibujo a cada lado del punto de fijación situado en el centro de la pantalla del ordenador (ver Figura 3B). Los resultados mostraron que los grupos sanos (adultos jóvenes y mayores sanos) presentaron un priming similar para los objetos atendidos. Como en los estudios anteriores, no se encontró priming para los objetos no atendidos. La memoria implícita no es automática y requiere atención. La actuación de los mayores con DCL fue similar a los EA y no mostraron facilitación para los objetos atendidos, lo que sugiere la existencia de un déficit temprano de la atención selectiva (Ballesteros et al., 2008). La falta de memoria implícita para los dibujos atendidos cuando se presenta 
simultáneamente otro dibujo no atendido podría ser un marcador de envejecimiento patológico.

\section{Neuroplasticidad, adaptación y entrenamiento para preservar la capacidad cognitiva de los mayores}

Estudios electroencefalográficos (Osorio et al., 2010; Sebastián y Ballesteros, 2012) y de resonancia magnética funcional recientes (Ballesteros, Bischof, Goh y Park, 2013; Park y Reuter-Lorenz, 2009) han mostrado la capacidad del cerebro para adaptarse a los cambios producidos durante el envejecimiento reclutando zonas adicionales para procesar la información y con un procesamiento neural más sostenido. Esta habilidad para adaptarse y posiblemente para producir tejido adicional es la base de la neuroplasticidad (Goh y Park, 2009). Los resultados de los estudios sobre atención y priming pueden tener implicaciones prácticas porque el DCL parece representar estadios previos de la EA y podría ser útil para detectar tempranamente la demencia. La realización de actividades estimulantes como la práctica de la danza (Kattenstroth, Kalisch, Holt, Tegenthoff y Dinse, 2013), el ejercicio físico (Voelcker-Rehage, Godde y Staudinger, 2010), el entrenamiento cognitivo con videojuegos (Ballesteros, Mayas, Toril et al., 2013; Ballesteros, Prieto, Mayas et al., 2014; Mayas, Parmentier, Andrés y Ballesteros, 2014) y la participación en actividades sociales que eviten el aislamiento y reduzcan el sentimiento de soledad e inseguridad mediante la utilización de las redes sociales y la utilización de nuevas tecnologías de la comunicación por ordenador como las desarrolladas en el proyecto Europeo AGNES (Ballesteros, Peter, Waterworth y Waterworth, 2012; Peter, Kreiner, Schröter et al., 2013) producen efectos beneficiosos y pueden contribuir a mantener la vitalidad cognitiva en la vejez, retrasando los efectos negativos del envejecimiento patológico.

\section{Reconocimientos}

La investigación revisada en la primera parte fue realizada en colaboración con el profesor Dionisio Manga, a cuyo reconocimiento está dedicado este Monográfico. La colaboración con el Dr. Manga data de los años ochenta del siglo pasado, cuando éramos profesores de la Facultad de Psicología de la Universidad Complutense de Madrid. Dicha colaboración continuó después cuando me trasladé a la UNED. La preparación de este artículo fue realizada con la ayuda del Ministerio de Ciencia e Innovación (PSI2010-21609-C02-01) y la Comunidad de Madrid (S2010/BDM-2349).

\section{Referencias}

Ballesteros, S. (1989). Some determinants of perceived structure: Effects of stimulus and tasks. En B. E. Shepp y S. Ballesteros (Eds.), Object perception: Structure and process (pp. 235-266). Hillsdale, NJ: Lawrence Erlbaum Associates.

Ballesteros, S., Bischof, G. N., Goh, J. O. y Park, D. C. (2013). Neural correlates of conceptual object priming in young and older adults: An eventrelated fMRI study. Neurobiology of Aging, 34, 1254-1264.

Ballesteros, S., González, M., Mayas, J., Reales, J. M., y García, B. (2009). Crossmodal object priming in young and older adults. European Journal of Cognitive Psychology, 21, 366-387.

Ballesteros, S., y Manga, D (1994). The influence of irrelevant information in visual perception. En S. Ballesteros (Ed.), Cognitive approaches to human perception (pp. 43-67). Hillsdale, NJ: Lawrence Erlbaum Associates.

Ballesteros, S. y Manga, D. (1996). The effects of variation of an irrelevant dimensión on samedifferent visual judgments. Acta Psychologica, 92, 1-16.

Ballesteros, S., Mayas, J. y Reales, J. M. (2013). Cognitive function in healthy aging and mild cognitive impaired older adults. Psicothema, 25, 18-24.

Ballesteros, S., Mayas, J., Toril, P., Prieto, A., Pita, C., Ponce de León, L. y Reales, J. M. (2013). The role of information computing technology, 
networking and videogame training on age related cognitive changes. Comunicación Invitada presentada en Cognition \& Aging, 2013 Congress, Dortmund, Alemania.

Ballesteros, A., Muñoz, F. y Reales, J. M. (2013). Stimulus fragmentation and the Stroop colorword interference: A behavioural and electrophysiological study. Comunicación presentada en la RECA 2013. Palma de Mallorca, España.

Ballesteros, S., Peter, C., Waterworth, E. L. y Waterworth, J. A. (2012). The role of ICT and networking in healthy aging. The 5th ACM International Conference on Pervasive Technologies Related to Assistive Environments: PETRA 2012 [CDROM]. New York: ACM, 2012.

Ballesteros, S., Prieto, A., Mayas, J., Toril, P., Pita, C., Ponce de León, L., Reales, J. M. y Waterworth, J. (2014 pending). Training older adults with non-action video games enhances cognitive functions that decline with aging: A randomized controlled trial. Frontiers in Aging Neuroscience.

Ballesteros, S. y Reales, J. M. (2004). Intact haptic priming in normal aging and Alzheimer's disease: Evidence for dissociable memory systems. Neuropsychologia, 44, 1063-1070.

Ballesteros, S., Reales, J. M. y García, B. (2007). The effects of selective attention on perceptual priming and explicit recognition in children with attention deficit and normal children. European Journal of Cognitive Psychology, 19, 607-627.

Ballesteros, S., Reales, J. M., García, E. y Carrasco, M. (2006). Selective attention effects on implicit and explicit memory for familiar objects at different delay conditions. Psicothema, 18, 96-107.

Ballesteros, S., Reales, J. M. y Manga, D. (2000). Effects of type of design (blocked vs. randomized) on Stroop and emotional Stroop tasks. Psicothema, 12, 60-63.

Ballesteros, S., Reales, J. M. y Mayas, J. (2007). Picture priming in aging and dementia. Psicothema, 19, 239-244.

Ballesteros, S., Reales, J. M., Mayas, J. y Heller, M. A. (2008). Selective attention modulates visual and haptic repetition priming: Effects on aging and Alzheimers' disease. Experimental Brain Research, 189, 473-483.
Braver, T. S. y West, R. (2008). Working memory, executive control and aging. En F. I. M. Craik y T. A. Salthouse (Eds.), The handbook of aging and cognition (3. ${ }^{a}$ Ed., pp. 311-372). New York, USA: Psychology Press.

Crabb, B. T. y Dark, V. J. (1999). Perceptual implicit memory requires attentional encoding. Memory and Cognition, 27, 267-275.

Craik, F. M. I., Goboni, R., Naveh-Benjamin, M., y Anderson, N. D. (1996). The effect of divided attention in encoding and retrieval processes in human memory. Journal of Experimental Psychology: General, 125,159-180.

Dixon, P. J. y Just, M. A. (1978). Normalization of irrelevant dimensions in stimulus comparisons. Journal of Experimental Psychology: Human Perception and Performance, 4, 36-46.

Fleischman, D. A. (2007). Repetition priming in aging and Alzheimer's disease: an integrative review and future directions. Cortex, 43, 889-897.

Garner, W. R. (1974). The processing of information and structure. Hillsdale, NJ: Erlbaum.

Goh, J. O., An, Y. y Resnick, S. M. (2012). Differential trajectories of age-related changes in components of executive and memory processes. Psychology and Aging, 27, 707-719.

Kattenstroth, J. C., Kalisch, T., Holt, S., Tegenthoff, M. y Dinse, H. R. (2013). Six months of dance intervention enhances postural, sensorimotor, and cognitive performance in elderly without affecting cardio-respiratory functions. Frontiers in Aging Neuroscience, 5, Article 6, 1-16.

Reisberg, D. (1997). Cognition: Exploring the science of mind. New York: Norton.

MacLeod, C. M. (1991). Half a century of research on the Stroop effect: An integrative review. Psychological Bulletin, 109, 163-203.

MacDonald, P. A. y MacLeod, C. M. (1998). The influence of attention at encoding in direct and indirect remembering. Acta Psychologica, 98, 291-310.

Mayas, J., Parmentier, F. B. R., Andrés, P. y Ballesteros, S. (2014). Plasticity of attentional functions in older adults after non-action video game training. PLoS ONE 9(3): e92269. doi:10.1371/ journal.pone.0092269. 
Mulligan, N. (1998). The role of attention during encoding on implicit and explicit memory. Journal of Experimental Psychology: Learning, Memory and Cognition, 24, 27-47.

Osorio, A., Fay, S., Pouthas, V. y Ballesteros, S. (2010). Ageing affects brain activity in highly educated older adults: An ERP study using a word-stem priming task. Cortex, 46, 522-534.

Park, D. C. y Reuter-Lorenz, P. (2009). The adaptative brain: Aging and neurocognitive scaffolding. Annual Review of Psychology, 60, 173-196.

Parkin, A. J. y Russo, R. (1990). Implicit and explicit memory and the automatic/effortful distinction. European Journal of Cognitive Psychology, 2, 71-80.

Peter, C., Kreisner, A., Schröter, M., Hyosun, K., Bieber, G., Öhberg, F., Hoshi, K., Lindh-Waterworth, E., Waterworth, J. y Ballesteros, S. (2013). AGNES: Connecting people in a multimodal way. Journal on Multimodal User Interfaces JMUI. doi: 10.1007/s12193-013-0118-z.

Reales, J. M. y Ballesteros, S. (1999). Implicit and explicit memory for visual and haptic objects: Cross-modal priming depends on structural descriptions. Journal of Experimental Psychology: Learning, Memory, and Cognition, 25, 644-663.

Rock, I. y Gutman, D. (1981). The effects of inattention on form perception. Journal of Experimental Psychology: Human Perception and Performance, 7, 27-287.
Sebastian, M. y Ballesteros, S. (2012). Effects of normal aging on event-related potentials and oscillatory brain activity during a haptic repetition priming task. NeuroImage, 60, 7-20.

Snodgrass, J. G. y Poster (1992). Visual world recognition thresholds for screen-fragmented words and Snodgrass and Vanderwart pictures. Behavioral Research Methods, Instruments and Computers, 24, 1-15.

Snodgrass, J. G. y Vanderwart, M. (1980). A stardardized set of 260 pictures: Norms for name agreement, image agreement, familiarity, and visual complexity. Journal of Experimental Psyhology: Human Learning and Memory, 6, 807-815.

Stroop, J. R. (1935). Studies on interference on serial verbal reactions. Journal of Experimental Psychology, 18, 643-662.

Verhaeghen, P. y Cerella, J. (2008). Everything we know about aging and response times: A meta-analytic integration. En S. Hofer y D. F. Alwin (Eds.), Handbook of cognitive aging: Interdisciplinary perspectives (pp.134-150). Los Angeles: CA: SAGE.

Voelcker-Rehage, C., Godde, B. y Staudinger, U. M. (2010). Physical and motor fitness are both related to cognition in old age. European Journal of Neuroscience, 31, 167-176.

Wiggs, C., Weisberg, J. y Martin, A. (2006). Repetition priming across the adult lifespan -the long and short of it. Aging, Neuropsychology and Cognition, 13, 308-25. 